\title{
IL-8 regulates the stemness properties of cancer stem cells in the small-cell lung cancer cell line H446
}

This article was published in the following Dove Press journal: OncoTargets and Therapy

Fang Jin ${ }^{1,2, *}$

Yajing Miao ${ }^{3, *}$

Pengyu $\mathrm{Xu}^{\prime}$

Xiaofei Qiu'

'Department of Pathology, Tianjin Medical University, Tianjin, China; ${ }^{2}$ Respiratory Department, Tianjin Medical University General Hospital, Tianjin, China; ${ }^{3}$ Research Center for Basic Medical Science, Tianjin Medical University, Tianjin, China

*These authors contributed equally to this work
Correspondence: Xiaofei Qiu Department of Pathology, Tianjin Medical University, No.22, Qixiangtai Road, Heping District, Tianjin 300070, China Tel +862260363l6I Email qiouxf@tmu.edu.cn
Purpose: Cancer stem cells (CSCs) are a small population of cancer cells located within a tumor that are highly tumorigenic, capable of tumor initiation, and resistant to cancer therapies. We identified the potential genes involved in regulating stemness properties and investigated the mechanisms in small-cell lung cancer (SCLC).

Materials and methods: Whole transcriptome sequencing technology was used to screen the potential genes involved in regulating stemness properties from SCLC-SCs $\left(\mathrm{uPAR}^{+}\right)$and differentiated cells $\left(\mathrm{uPAR}^{-}\right)$in the $\mathrm{H} 446$ cell line. The selected genes were validated by quantitative reverse transcription PCR and ELISAs. The effect of IL-8 on stemness of sphere-forming cells was determined through tumor sphere formation, wound healing migration, and in vivo tumorigenesis assays.

Results: In our study, $\mathrm{uPAR}^{+}$and $\mathrm{uPAR}^{-}$cells showed different gene expression profiles. IL-8 was upregulated in SCLC sphere-forming cells. Blocking IL-8 expression with siRNA led to loss of stemness, including the self-renewal capability, migration, expression of stemness-related genes, and in vivo tumorigenicity, in sphere-forming cells. Consistently, exogenously added IL-8 enhanced stemness properties in parental cells.

Conclusion: IL-8 was upregulated in SCLC sphere-forming cells, and critical for the acquisition and/or maintenance of the stemness features in the SCLC cell line H446. Our results suggest that blocking IL-8 signaling may provide a novel therapeutic approach for targeting SCLC-SCs and improve treatment and outcomes in SCLC.

Keywords: small-cell lung cancer, cancer stem cells, tumor sphere, IL-8, uPAR, stemness

\section{Introduction}

Small-cell lung cancer (SCLC) is one of the most aggressive lung cancers and has a poor prognosis, as symptoms are generally nonspecific, and $70 \%$ of patients are already in stage IIIb or IV at the time of diagnosis. ${ }^{1}$ Therefore, systemic (platinum-based) chemotherapy remains the most common strategy for first-line treatment. Although patients initially respond well to treatment, resistance to chemotherapy inevitably develops. Cancer stem cells (CSCs) are thought to be responsible for tumor initiation and maintenance, therapy resistance, and tumor relapse. The drug resistance in SCLC could be attributable to the existence of CSCs in SCLC. Therefore, targeting of SCLCSCs is important for the treatment of SCLC. A better understanding of the molecular biology of SCLC will lead to advances in the treatment of SCLC. ${ }^{2}$

There is a close relationship between the cytokines in the tumor microenvironment and the occurrence and development of tumors. ${ }^{3}$ In head and neck squamous 
cell carcinomas, tumor-associated endothelial cell-secreted IL-6 defined the tumorigenic potential of CSCs, which enhanced orosphere formation, p-STAT3 activation, survival, and self-renewal of human CSCs. ${ }^{4}$ IL-17 promoted the self-renewal and tumorigenesis capacity of ovarian $\mathrm{CD}_{133^{+}}$cancer stem-like cells. ${ }^{5}$ In human colon CSCs, IL-12 reduced invasiveness, tumorsphere formation, and tumor initiating capacity by reducing the expression of IL-4 and STAT6 in CSCs. ${ }^{6}$ IL-8 also increased stem-like properties, including aldehyde dehydrogenase activity, clonogenic activity, and in vivo tumorigenicity, in lung cancer cells. ${ }^{7}$ CXCR1 expression was positively correlated with the CSC markers CD44 and CD133, and further functional experiments confirmed that the IL-8/CXCR1 axis was related to the characteristics of pancreatic CSCs. ${ }^{8}$ IL-8 regulates the breast CSC activity through its receptors CXCR1 and CXCR2. A CXCR1/2 inhibitor combined with HER2-targeted therapy can eliminate CSCs and improve survival in patients with breast cancer. ${ }^{9}$ These data suggest that cytokines are associated with properties of CSCs and treatment resistance.

Previous work from our laboratory showed that tumor spheres from SCLC cell lines were characterized by stemlike properties, and stem-like cells were enriched after consecutive passaging in a defined serum-free medium. Furthermore, the stem-like cell population may be enriched in cells expressing the urokinase plasminogen activator receptor (UPAR) cell surface marker. ${ }^{10}$ Therefore, we used uPAR sorting to enrich CSCs in the SCLC cell line H446. In our study, we undertook a systematic comparison of genes in $\mathrm{uPAR}^{+}$cells and $\mathrm{uPAR}^{-}$cells. Our results showed robust upregulation of $I L-8$ in the $\mathrm{uPAR}^{+}$cells compared with $\mathrm{uPAR}^{-}$cells. Blockade of IL-8 signaling with siRNA led to loss of stemness, including self-renewal capability, migration, expression of stemness-related genes, and in vivo tumorigenicity in SCLC-SCs. The human recombinant IL-8 markedly enhanced the stemness properties in parental cells. These results thus provide an experimental basis for the development of new therapeutic approaches involving blockade of IL-8 signaling in SCLC patients.

\section{Materials and methods}

\section{Cell line and cell culture}

The SCLC cell line H446 was obtained from the American Type Culture Collection (ATCC) and grown in the culture medium and culture conditions recommended by the ATCC. The procedure for obtaining tumor sphere cell culture was described in our previous study. ${ }^{10}$

\section{RNA, cDNA, and quantitative reverse transcription PCR (qRT-PCR)}

Total RNA was isolated from cells using the TRIzol reagent RNA isolation method. A total of $1.0 \mu \mathrm{g}$ of extracted RNA was used to synthesize cDNA using a FastQuant RT kit (Tiangen Biotech [Beijing] Co. Ltd, Beijing, China). The primer sequences (Table 1) were found in PrimerBank. The reactions were conducted by heat denaturing at $95^{\circ} \mathrm{C}$ for $15 \mathrm{~min}$, then 40 cycles of $95^{\circ} \mathrm{C}$ for $10 \mathrm{~s}$ followed by $57^{\circ} \mathrm{C}$ for $20 \mathrm{~s}$, and finally $72^{\circ} \mathrm{C}$ for $30 \mathrm{~s}$. RNA expression was measured by the cycle threshold (CT) value. The second derivative of the CT value was determined using the CT value determination method to compare fold differences.

\section{ELISAs}

Levels of IL-8 and IL-2 in the culture medium were determined by commercially obtained ELISA kits (Boster Biological Technology, Ltd, Fremont, CA, USA) according to the manufacturer's instructions.

\section{Cell transfection}

IL-8 siRNA (silL-8) and its negative control oligonucleotide (siNC) were obtained from RiboBio, Co, Ltd (Guangzhou, China). The transfection was performed using ribo $F E C T^{\text {тм}}{ }^{\text {E}}$ CTe perform (RiboBio, Co, Ltd) according to

Table I Quantitative reverse transcription PCR primer sequences

\begin{tabular}{ll}
\hline Primer name & Sequence \\
\hline GAPDH & Forward: CCATGTTCGTCATGGGTGT \\
& Reverse: TGAGTCCTTCCACGATACC \\
CDI33 & Forward: AGTCGGAAACTGGCAGATAGC \\
& Reverse: GGTAGTGTTGTACTGGGCCAAT \\
CD44 & Forward: CTGCCGCTTTGCAGGTGTA \\
& Reverse: CATTGTGGGCAAGGTGCTATT \\
SOX2 & Forward: GCCGAGTGGAACTTTTGTCG \\
& Reverse: GGCAGCGTGTACTTATCCTTCT \\
NANOG & Forward: TTTGTGGGCCTGAAGAAAACT \\
& Reverse: AGGGCTGTCCTGAATAAGCAG \\
uPAR & Forward: CCGGGAGAGATAGTGTACCCCTT \\
& Reverse: CAGCTCAGTATTGCAGCAGGA \\
OCT4 & Forward: CTGGGTTGATCCTCGGACCT \\
& Reverse: CCATCGGAGTTGCTCTCCA \\
IL-8 & Forward: GGTGCAGTTTTGCCAAGGAG \\
& Reverse: TTCCTTGGGGTCCAGACAGA \\
CXCL2 & Forward: AGTGTGTGGTCAACATTTCTCA \\
& Reverse: GCTCTAACACAGAGGGAAACAC \\
SATI & Forward: TGCCGCACTCAGTGTTGTTAG \\
& Reverse: GCAATTCCCGCACAAGATTCT \\
SPINK6 & Forward: TGACTGTGGTGAGTTCCAGGA \\
& Reverse: CCACTTTTCACTATGGCCTTACA \\
IGFBP3 & Forward: AGAGCACAGATACCCAGAACT \\
& Reverse: GGTGATTCAGTGTGTCTTCCATT \\
\hline
\end{tabular}


the instructions provided by the manufacturer. Briefly, cells were trypsinized, counted, and seeded in plates the day before transfection to ensure a suitable cell confluence on the day of transfection. silL-8 or siNC was transfected into H446 cells at a final concentration of $30 \mathrm{nM}$. Transfection efficiency was monitored by qRT-PCR.

\section{Tumor sphere formation assay}

After transfection with $30 \mathrm{nM}$ of silL-8 or siNC, cells were trypsinized, counted, and seeded for tumor sphere formation assays in a six-well ultra low attachment plate at a density of 5,000 cells per well. The tumor sphere culture was performed as mentioned above. The total number of tumor spheres was counted after 6 days.

H446 cells were seeded into six-well ultra low attachment plates at a density of 5,000 cells per well. The experimental groups were treated with $10 \mathrm{ng} / \mathrm{mL}$ human recombinant IL-8 (R\&D Systems, Inc., Minneapolis, MN, USA). The total number of tumor spheres was counted after 6 days.

\section{Wound healing migration assay}

H446 cells were seeded into six-well plates at a density of $1 \times 10^{5}$ cells per well. At $24 \mathrm{~h}$ after seeding, the confluent monolayer of the culture was scratched with a pipette tip. Then, the cells were incubated at $37^{\circ} \mathrm{C}$ and photographed at 0 and $48 \mathrm{~h}$ on a microscope.

\section{In vivo tumorigenesis assay}

All protocols were approved by the Institutional Animal Care and Use Committee of the Institute of Hematology and Blood Disease Hospital, Chinese Academy of Medical Sciences and carried out according to institutional guidelines. Fiveweek-old nude mice (BALB/c-nu, female) were housed in a sterile facility and maintained in a standard temperature- and light-controlled animal facility. SCLC sphere-derived cells $\left(1 \times 10^{6}\right.$ cells $)$ were subcutaneously injected into nude mice. Tumor growth was monitored visually every week. Once the average size of tumor reached approximately $80 \mathrm{~mm}^{3}$, the mice were randomized into two groups $(n=6)$ and treated with siIL-8 or siNC. For delivery of siRNA, 1 nmol RNA in $0.1 \mathrm{~mL}$ PBS solution was locally injected into the tumor mass once every 4 days. The size of each tumor was measured once every 2 days and the volume of the tumor was calculated with the following formula: $V=a \times b^{2} / 2$, where $a$ is the long diameter and $b$ is the short diameter. Mice were sacrificed on day 20 after intratumoral injections, and tumors were photographed and harvested for analysis.

\section{Statistical analysis}

Statistical analysis was performed using SPSS18.0 software. Each experiment was performed in at least three independent trials. All data from three independent experiments are expressed as the mean \pm standard error of the mean. Statistical differences were determined by Student's $t$-test. $P<0.05$ was considered statistically significant.

\section{Results \\ IL-8 was identified as a potential chemokine for regulating CSCs in the SCLC cell line $\mathrm{H} 446$ through genetic screens}

Our previous study showed that the $\mathrm{PAAR}^{+}$cells in the SCLC cell line $\mathrm{H} 446$ were enriched for CSCs. ${ }^{10}$ To identify potential genes involved in regulating stem cell properties of SCLC, $\mathrm{uPAR}^{+}$and $\mathrm{uPAR}^{-}$cells were subjected to RNA-seq analysis, which showed different gene expression profiles. Then, we selected significantly upregulated genes in $\mathrm{UPAR}^{+}$cells compared with $\mathrm{UPAR}^{-}$cells by the Genes and Genomes database (KEGG) and identified five genes, including CXCL2, SAT1, IL-8, SPINK6, and IGFBP3 (Table 2). Previous work from our laboratory showed that tumorspheres from the SCLC cell line H446 were characterized by stem-like properties, and the third-passage sphere cells could serve as a high-purity CSC-like model. ${ }^{10}$ Therefore, the sphere cells were used for further analysis. The selected genes were validated in one set of H446 third-passage sphere cells (CSC populations) and adherent cells (non-CSC populations) by qRT-PCR. The expression values were normalized to the $G A P D H$ gene. As shown in Figure 1A, qRT-PCR analysis confirmed that the levels of $I L-8$ and $C X C L 2$ were significantly higher in sphere cells than adherent cells. Using ELISAs, we measured the levels of IL-8 and CXCL2 in culture media of sphere cells and adherent cells. We found that the IL-8 level was higher in sphere-derived culture media than in culture media from adherent cells (Figure 1B). However, the CXCL2 level

Table 2 Differentially expressed genes between $\mathrm{UPAR}^{+}$cells and uPAR $^{-}$cells in SCLC cell line H446

\begin{tabular}{ll}
\hline Gene name & Fold change \\
\hline CXCL2 & 3.42 \\
SATI & 2.74 \\
IGFBP3 & 2.72 \\
IL-8 & 3.47 \\
SPINK6 & 2.10 \\
\hline
\end{tabular}

Abbreviations: SCLC, small-cell lung cancer; uPAR, urokinase plasminogen activator receptor. 

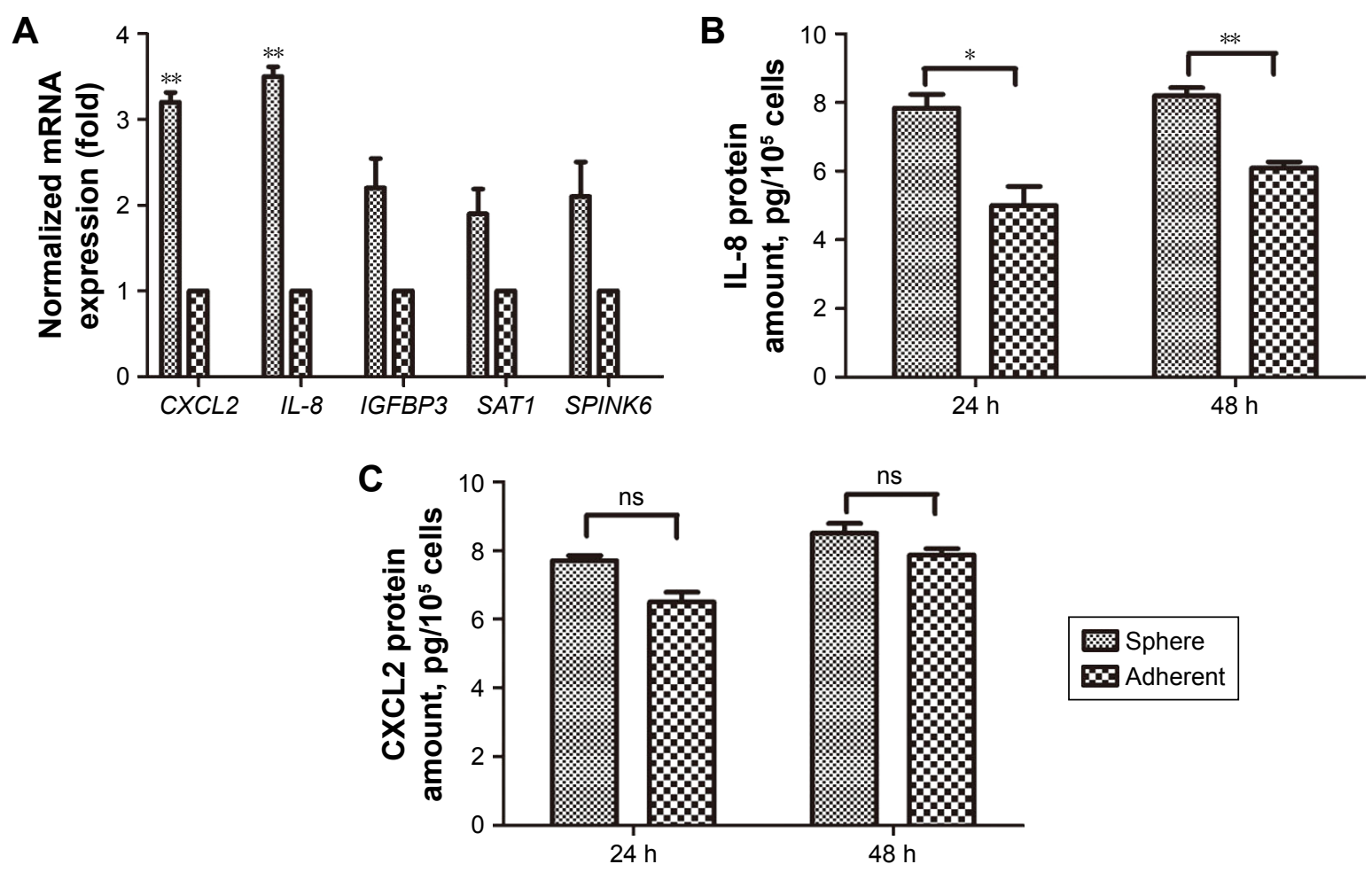

Figure I Upregulation of IL-8 in sphere cells.

Notes: (A) The expression levels of selected genes were determined in sphere cells and adherent cells from the H446 cell line by qRT-PCR. For each target gene, expression levels are calculated relative to the expression levels in adherent cells, arbitrarily set as I. Each qRT-PCR assay was performed at least three times. $* * P<0.0$ I. (B) IL-8 secretion and (C) CXCL2 secretion by sphere cells and adherent cells were analyzed after $24 \mathrm{~h}$ and $48 \mathrm{~h}$ by ELISAs. Data are expressed as the mean \pm standard error of the mean of three independent experiments. $* P<0.05, * * P<0.01$.

Abbreviations: ns, not significant; qRT-PCR, quantitative reverse transcription PCR.

showed no significant difference (Figure 1C). Therefore, we proposed that IL-8 might be involved in regulating the stemness of CSCs in the H446 cell line.

\section{Effect of IL-8 on the self-renewal capability of CSCs in the H446 cell line}

To evaluate the role of IL-8 in maintaining the stemness property of CSCs in the H446 cell line, we used the RNA interference technology to silence IL-8 expression in the tumor spheres and verified the IL-8 expression by qRT-PCR and ELISAs. The results showed that the mRNA and protein levels of IL-8 were significantly inhibited in the silL- 8 treated tumorspheres, which indicated that IL-8 was silenced successfully in tumorspheres (Figure 2A and B). Then, tumor sphere formation assays were performed to investigate the effect of IL-8 on self-renewal. The results showed that the cells treated with silL-8 formed fewer tumor spheres than those treated with negative control siNC (Figure 2C and D). Meanwhile, the tumor sphere size was smaller in silL-8 cells than siNC cells. Furthermore, we investigated the effect of exogenous IL-8 on self-renewal in the H446 cell line. The cells treated with human recombinant IL-8 (rh-IL-8) formed significantly more and larger tumor spheres than the untreated group (Figure 2E and F). Therefore, IL-8 enhanced the self-renewal ability of parental cells. These results suggest that IL-8 plays a crucial role in self-renewal of CSCs in vitro.

\section{Effect of IL-8 on stemness-associated genes of CSCs in the H446 cell line}

There are many CSC factors that are overexpressed in CSC populations. To further determine the effect of IL-8 on stemness marker expression, we analyzed the stemness-related genes expression after knockdown of IL-8 by qRT-PCR. The results showed that stemness-related OCT4, CD133, and Nanog were significantly suppressed in tumorspheres treated by silL- 8 compared with control siNC cells. However, the expression of $C D 44$ in the two groups had no significant difference (Figure 3A). To investigate the effect of exogenous IL- 8 on stemness-related genes expression, we further detected the expression of $u P A R, C D 44, S O X 2$, and Nanog after the rh-IL-8 treatment of adherent cells. The results showed that the $u P A R, C D 44$, SOX2, and Nanog expression 
A

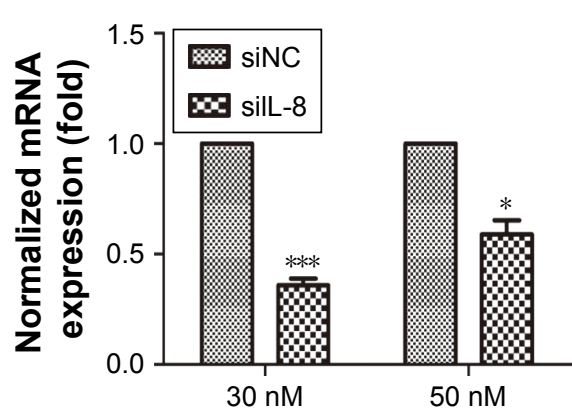

B

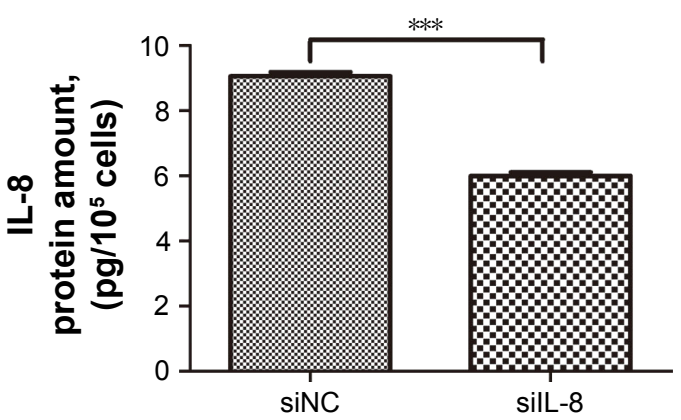

C

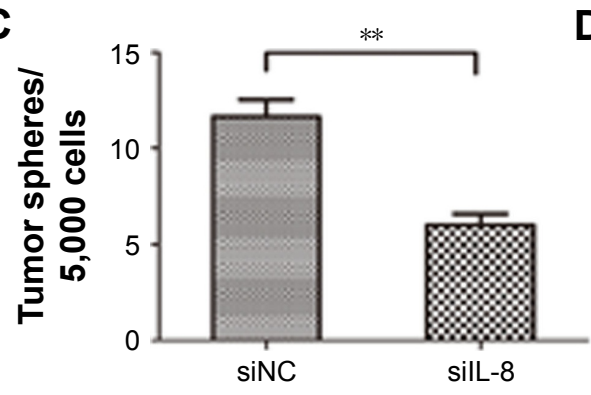

E

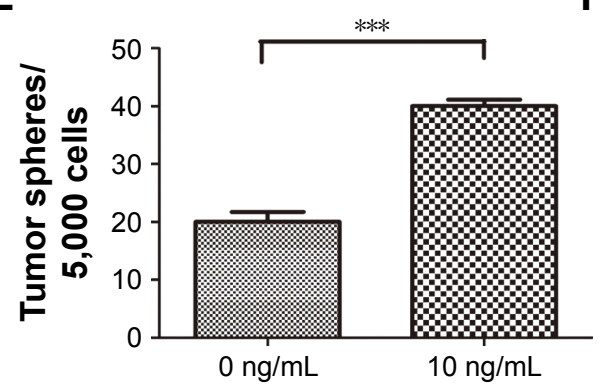

D

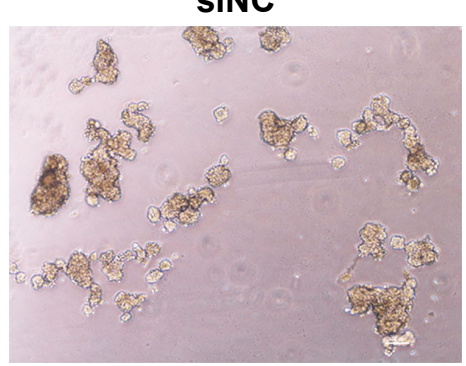

$0 \mathrm{ng} / \mathrm{mL}$

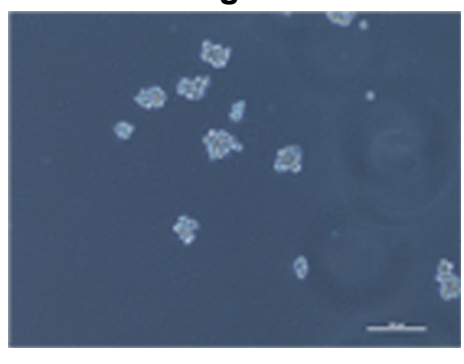

silL-8

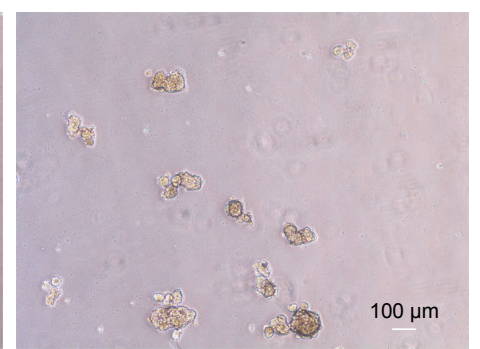

$10 \mathrm{ng} / \mathrm{mL}$

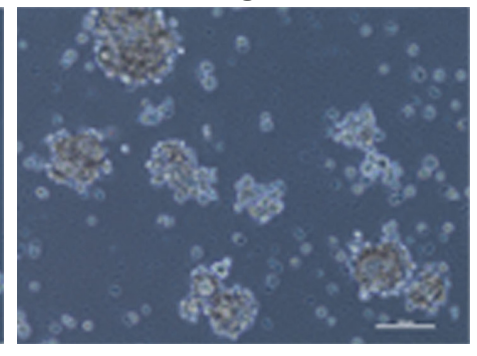

Figure 2 The effect of IL-8 on self-renewal of CSCs in the H446 cell line.

Notes: (A) The expression of IL-8 in sphere cells transfected with silL- 8 or negative control (siNC) was detected by qRT-PCR. $* P<0.05$, $* * * P<0.00$, compared with control. (B) IL-8 secretion by sphere cells transfected with silL-8 or siNC was analyzed by ELISAs. Data are expressed as the mean \pm standard error of the mean (sem) of three independent experiments. $* * * P<0.00 I$. (C) The sphere formation capability was evaluated in sphere cells transfected with silL-8 or siNC. Data are expressed as the mean \pm sem of three independent experiments. $* * P<0.0$ I. (D) Representative micrographs of spheres formed from cells transfected with silL-8 or siNC. Scale bar I00 $\mu$ m. (E) The sphere formation capability of $\mathrm{H} 446$ cells treated with $0 \mathrm{ng} / \mathrm{mL}$ or $10 \mathrm{ng} / \mathrm{mL}$ human recombinant IL-8 (rh-IL-8). Data are expressed as the mean \pm sem of three independent experiments. $* * * P<0.00 \mathrm{I}$. (F) Representative micrographs of spheres formed by $\mathrm{H} 446$ cells treated or not with rh-IL-8. Scale bar $50 \mu \mathrm{m}$.

Abbreviations: silL-8, IL-8 siRNA; siNC, negative control oligonucleotide.
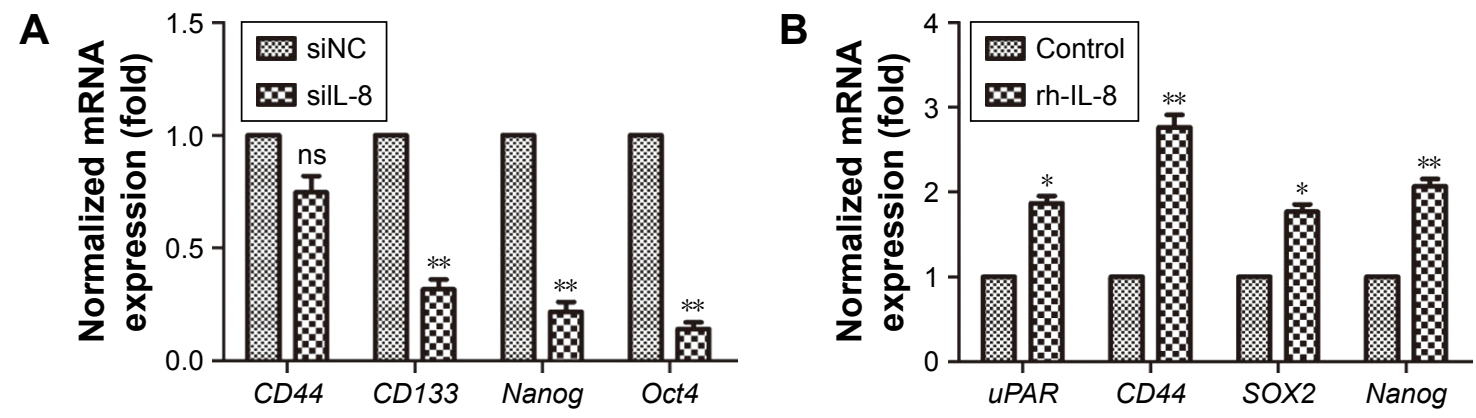

Figure 3 The effect of IL-8 on expression of stemness genes.

Notes: (A) The levels of stemness-related genes were analyzed in sphere cells transfected with silL- 8 or siNC by qRT-PCR. $* * P<0.01$, compared with control. (B) The mRNA levels of the indicated stemness-related genes in $\mathrm{H} 446$ cells treated with $100 \mathrm{ng} / \mathrm{mL}$ rh-IL-8. Data are expressed as mean \pm standard error of the mean of three independent experiments. $* P<0.05$, $* * P<0.01$, compared with control.

Abbreviations: ns, not significant; rh-IL-8, human recombinant IL-8; silL-8, IL-8 siRNA; siNC, negative control oligonucleotide; uPAR, urokinase plasminogen activator receptor; qRT-PCR, quantitative reverse transcription PCR. 
was higher in rh-IL-8 treated cells than the untreated group (Figure 3B). These data suggest that IL-8 could confer the stemness of CSCs in the H446 cell line.

\section{IL-8 enhances the migration capacity of $\mathrm{H} 446$ cells in vitro}

Next, to analyze the effect of IL-8 on other CSC phenotypes, such as cell migration, we performed in vitro wound healing assays. We treated the $\mathrm{H} 446$ cells with $10 \mathrm{ng} / \mathrm{mL}$ rh-IL-8 and terminated the experiment after $48 \mathrm{~h}$. The results showed that cells treated with rh-IL-8 had an increased migratory ability after $48 \mathrm{~h}$, which led to a significant reduction in the width of the wound compared with the control (Figure 4A and B). These data further validated the role of IL-8 in increasing CSC phenotypes in H446 cells.

\section{silL-8 treatment inhibits the growth of xenografts}

To investigate the effect of silL- 8 treatment on tumor growth in vivo, we used tumorsphere cells for generation of tumor xenografts. When the tumor size was $\sim 80 \mathrm{~mm}^{3}$, we injected silL- 8 every 4 days into the tumor mass, and the volume of tumor was calculated every 2 days. The results showed that for each xenotransplant, the tumor growth is significantly inhibited by treatment with silL- 8 , with a $20 \%$ decrease in mean tumor volume on day 20 (Figure 5A and B).

\section{Discussion}

In recent years, both the standard chemotherapy (platinumetoposide) and the median survival time have not significantly changed for SCLC. The drug resistance and tumor relapse

\section{A}

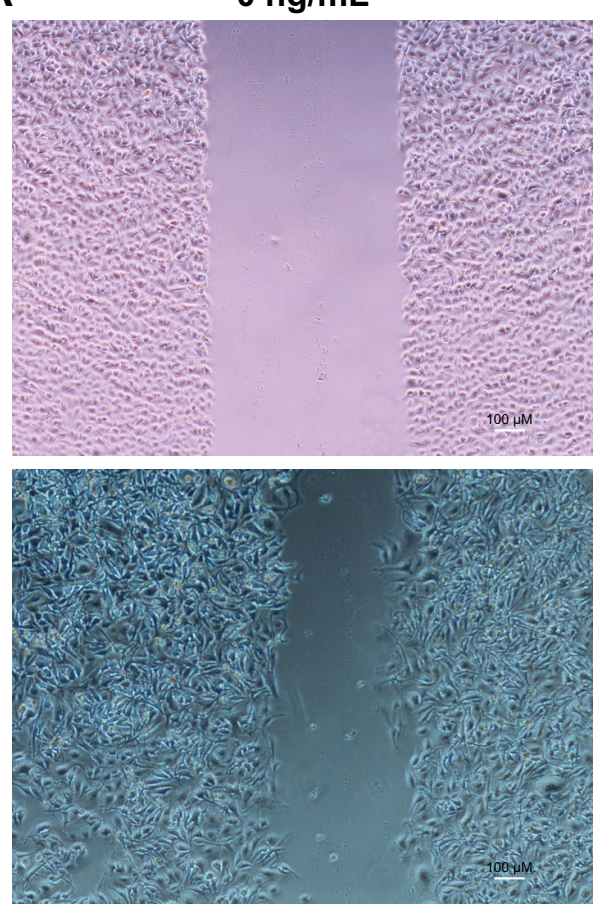

\section{$10 \mathrm{ng} / \mathrm{mL}$}
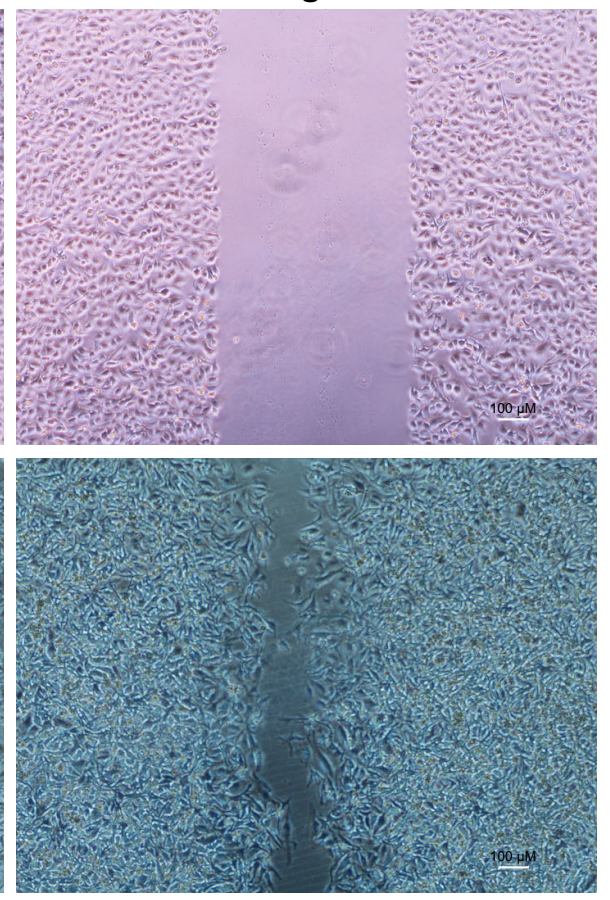

B

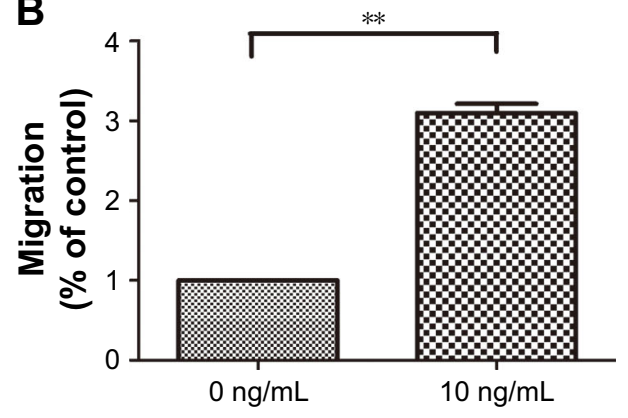

Figure 4 IL-8 increases the migration of H446 cells.

Notes: (A) The wound healing assay indicated that human recombinant IL-8 (rh-IL-8) treated H446 cells had a significant reduction in the width of the wound compared with the control after $48 \mathrm{~h}$. (B) The migration distances of $\mathrm{H} 446$ cells treated by $10 \mathrm{ng} / \mathrm{mL}$ rh-IL-8 are shown. Data are expressed as mean \pm standard error of the mean of three independent experiments. $* * P<0.01$. 


\section{A}
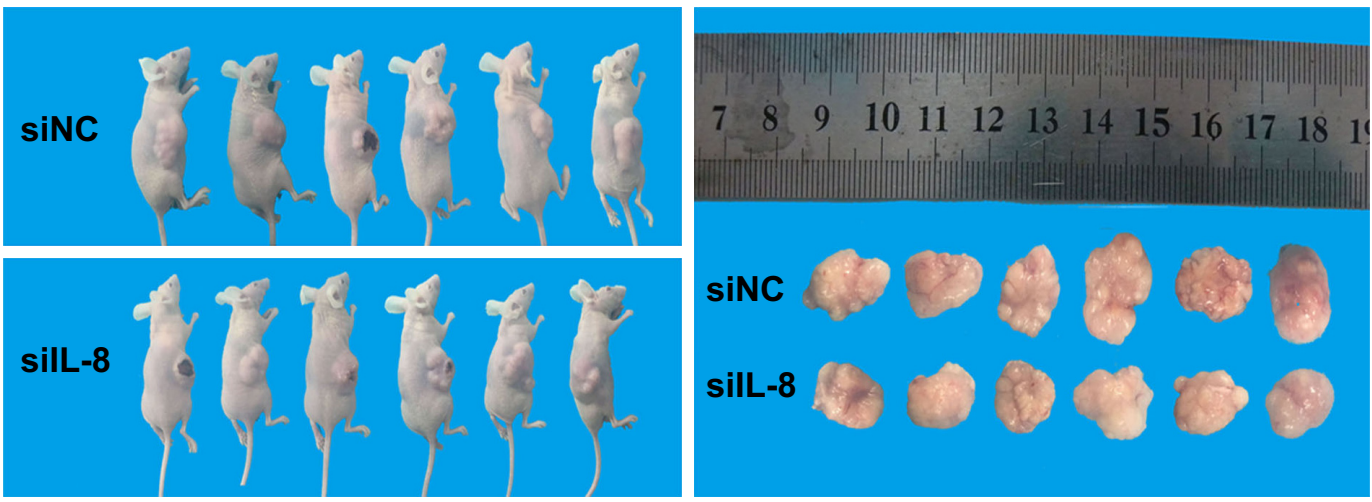

B

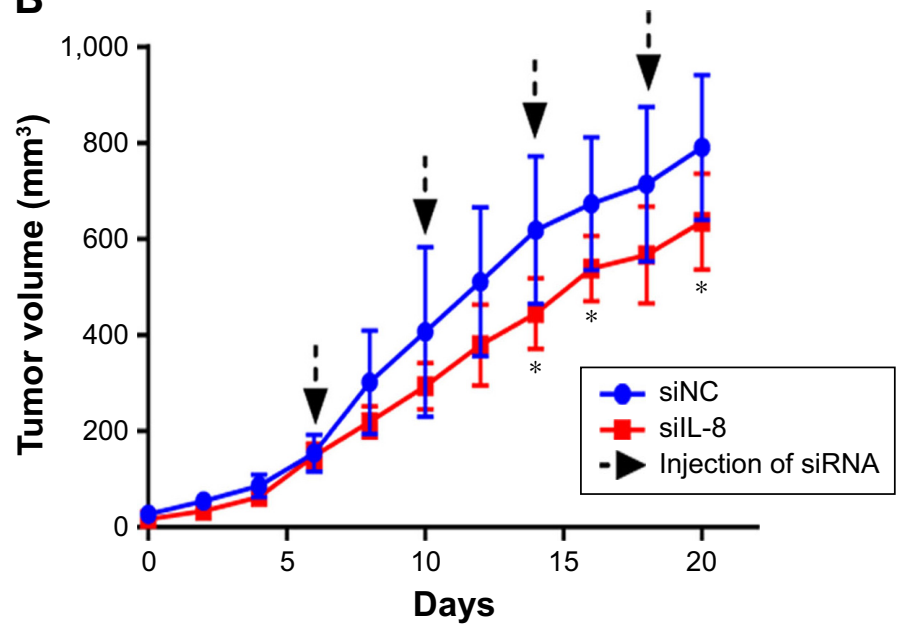

Figure 5 silL-8 treatment inhibits tumor growth.

Notes: (A) Subcutaneous implantation of sphere cells in nude mice led to tumor formation. (B) Growth curve of xenograft tumors volume of silL-8 treatment group and siNC control group. $* P<0.05$.

Abbreviations: silL-8, IL-8 siRNA; siNC, negative control oligonucleotide.

in SCLC could be attributable to the existence of CSCs in SCLC. In the present study, IL-8 was shown to be critical for the acquisition and/or maintenance of the stemness features in the SCLC cell line H446. The association between inflammation and cancer is well established, and deregulated expression of multiple inflammatory cytokines, including IL-8, in malignant diseases is well recognized. ${ }^{11}$ IL-8/CXCL8 belongs to the CXC chemokine family and was originally identified as a neutrophil chemoattractant with inflammatory activity. ${ }^{12,13}$ IL-8 exerts its biological effect by binding to its receptors, which are two heterotrimeric $\mathrm{G}$ protein-coupled receptors, CXCR1 and CXCR2. ${ }^{14,15}$ CXCR1 and CXCR2 have been found on many normal cells, such as neutrophils, basophils, lymphocytes, monocytes, keratinocytes, and endothelial cells. ${ }^{16}$ In healthy tissues, IL-8 is almost undetectable, but it is rapidly induced by 10 - to 100 -fold in response to proinflammatory cytokines, such as tumor necrosis factor or IL-1, bacterial or viral products, and cellular stress. ${ }^{17}$ Cooption of IL-8/IL-8R is now known to be an established occurrence in human cancer and has been shown to promote tumor progression by multiple means. ${ }^{18}$ IL- 8 expression has been detected in numerous cancer types, including breast cancer, prostate cancer, lung cancer, colorectal carcinoma, and melanoma. ${ }^{11}$ Furthermore, the IL- 8 level in cell line culture medium depended on the cell type. ${ }^{16,19}$

Elevated IL-8 was detected in lung cancer, especially in non-small-cell lung cancer (NSCLC) cell lines. ${ }^{16,20}$ IL-8 has been found to promote cell proliferation, ${ }^{21}$ invasion and metastasis, ${ }^{22}$ and drug resistance. ${ }^{7,23}$ IL-8 expression is an unfavorable prognostic factor, and high IL-8 mRNA expression correlates with tumor progression, tumor angiogenesis, shortened survival time, and early relapse in NSCLC..$^{24,25}$ Moreover, a high plasma IL-8 level was correlated with shorter progression-free-survival time in NSCLC. ${ }^{7}$ IL-8 can confer resistance to EGFR inhibitors by inducing stem cell properties and acquisition and/or maintenance of mesenchymal traits. ${ }^{7,23}$ Autocrine CXCL8 and VEGF collaboratively mediate neovascularization 
and epithelial-mesenchymal transition, which facilitates invasion in A549 cells. $^{26}$

Although IL-8 has been found to play multiple roles in cancer development, the mechanisms by which IL-8 contributes to SCLC progression have remained poorly understood. A few papers have examined IL-8 expression and studied the role in non-CSCs in SCLC cell lines. ${ }^{16,19}$ However, recent studies indicated that IL-8 may drive tumorigenesis by promoting CSC invasion, metastasis, self-renewal, and treatment resistance. ${ }^{7,27,28}$ In our study, IL-8 was upregulated in sphere-forming cells. Furthermore, we investigated the role of IL- 8 in stemness properties in the H446 cell line. Our results showed that IL-8 is capable of driving stem-like properties in SCLC cells. Knockdown of IL-8 expression with siRNA led to loss of stemness, including self-renewal capability, migration, expression of stemness-related genes, and in vivo tumorigenicity, in sphere-forming cells. In contrast, exogenously increasing IL-8 in parental cells enhanced the stem-like properties of SCLC cells in vitro and converted less malignant cells into highly malignant cells. These data suggested that IL- 8 is critical for the acquisition and/or maintenance of the stemness features in the SCLC cell line H446. Our results suggest that blocking IL- 8 signaling may provide a novel therapeutic approach for targeting SCLC-SCs and improve treatment and outcomes in SCLC.

Several studies and our data have shown the influence of IL- 8 in driving the CSC phenotype. Moreover, the IL- 8 receptor CXCR $1 / 2$ is also specifically overexpressed in breast CSCs compared with bulk tumor cells, and these cells are resistant to Fas ligand-induced apoptosis. ${ }^{29}$ Importantly, a CXCR1 inhibitor, repertaxin, was able to specifically target the CSC population in human breast cancer xenografts, synergize with chemotherapy agents to retard tumor growth, and inhibit metastases. Because of the important role of CXCR1/2 in IL-8/CXCR1/2 signaling, our laboratory will further investigate the effect of CXCR $1 / 2$ on SCLC-SCs and its prognostic value in human SCLC.

\section{Acknowledgment}

This work was supported by the Research Program of the Applied Basic and Cutting-edge Technologies of Tianjin under contract no 14JCZDJC35500.

\section{Disclosure}

The authors report no conflicts of interest in this work.

\section{References}

1. Fujita J, Saijo N. Current perspectives in the management of small cell lung cancer. Gan No Rinsho. 1985;31(1):1-19.
2. Kurup A, Hanna NH. Treatment of small cell lung cancer. Crit Rev Oncol Hematol. 2004;52(2):117-126.

3. Cheng ZH, Shi YX, Yuan M, Xiong D, Zheng JH, Zhang ZY. Chemokines and their receptors in lung cancer progression and metastasis. J Zhejiang Univ Sci B. 2016;17(5):342-351.

4. Krishnamurthy S, Warner KA, Dong Z, et al. Endothelial interleukin-6 defines the tumorigenic potential of primary human cancer stem cells. Stem Cells. 2014;32(11):2845-2857.

5. Xiang $\mathrm{T}$, Long $\mathrm{H}, \mathrm{He} \mathrm{L}$, et al. Interleukin-17 produced by tumor microenvironment promotes self-renewal of CD133+ cancer stem-like cells in ovarian cancer. Oncogene. 2015;34(2):165-176.

6. Yin XL, Wang N, Wei X, Xie GF, Li JJ, Liang HJ. Interleukin-12 inhibits the survival of human colon cancer stem cells in vitro and their tumor initiating capacity in mice. Cancer Lett. 2012;322(1):92-97.

7. Liu YN, Chang TH, Tsai MF, et al. IL-8 confers resistance to EGFR inhibitors by inducing stem cell properties in lung cancer. Oncotarget. 2015;6(12):10415-10431.

8. Chen L, Fan J, Chen H, et al. The IL-8/CXCR1 axis is associated with cancer stem cell-like properties and correlates with clinical prognosis in human pancreatic cancer cases. Sci Rep. 2014;4:5911.

9. Singh JK, Simões BM, Howell SJ, Farnie G, Clarke RB. Recent advances reveal IL-8 signaling as a potential key to targeting breast cancer stem cells. Breast Cancer Res. 2013;15(4):210.

10. Qiu X, Wang Z, Li Y, Miao Y, Ren Y, Luan Y. Characterization of sphere-forming cells with stem-like properties from the small cell lung cancer cell line H446. Cancer Lett. 2012;323(2):161-170.

11. Liu Q, Li A, Tian Y, et al. The CXCL8-CXCR1/2 pathways in cancer. Cytokine Growth Factor Rev. 2016;31:61-71.

12. Baggiolini M, Walz A, Kunkel SL. Neutrophil-activating peptide-1/ interleukin 8, a novel cytokine that activates neutrophils. J Clin Invest. 1989;84(4):1045-1049.

13. Schröder JM, Mrowietz U, Morita E, Christophers E. Purification and partial biochemical characterization of a human monocyte-derived, neutrophil-activating peptide that lacks interleukin 1 activity. J Immunol. 1987;139(10):3474-3483.

14. Holmes WE, Lee J, Kuang WJ, Rice GC, Wood WI. Structure and functional expression of a human interleukin-8 receptor. Science. 1991;253:1278-1280.

15. Murphy PM, Tiffany HL. Cloning of complementary DNA encoding a functional human interleukin-8 receptor. Science. 1991;253: $1280-1283$.

16. Zhu YM, Webster SJ, Flower D, Woll PJ. Interleukin-8/CXCL8 is a growth factor for human lung cancer cells. Br J Cancer. 2004; 91(11):1970-1976.

17. Hoffmann E, Dittrich-Breiholz O, Holtmann H, Kracht M. Multiple control of interleukin-8 gene expression. J Leukoc Biol. 2002;72(5): 847-855.

18. David JM, Dominguez C, Hamilton DH, Palena C. The IL-8/IL-8R Axis: A Double Agent in Tumor Immune Resistance. Vaccines. 2016;4(3): E22:22.

19. Niiya M, Niiya K, Kiguchi T, et al. Induction of TNF-alpha, uPA, IL-8 and MCP-1 by doxorubicin in human lung carcinoma cells. Cancer Chemother Pharmacol. 2003;52(5):391-398.

20. Khan MN, Wang B, Wei J, et al. CXCR1/2 antagonism with CXCL8/ Interleukin-8 analogue CXCL8(3-72)K11R/G31P restricts lung cancer growth by inhibiting tumor cell proliferation and suppressing angiogenesis. Oncotarget. 2015;6(25):21315-21327.

21. Hattar K, Reinert CP, Sibelius U, et al. Lipoteichoic acids from Staphylococcus aureus stimulate proliferation of human non-small-cell lung cancer cells in vitro. Cancer Immunol Immunother. 2017;66(6):799-809.

22. Zhang J, Chang L, Jin H, et al. Benzopyrene promotes lung cancer A549 cell migration and invasion through up-regulating cytokine IL8 and chemokines CCL2 and CCL3 expression. Exp Biol Med. 2016;241(14): 1516-1523.

23. Fernando RI, Hamilton DH, Dominguez C, David JM, Mccampbell KK, Palena C. IL-8 signaling is involved in resistance of lung carcinoma cells to erlotinib. Oncotarget. 2016;7(27):42031-42044. 
24. Yuan A, Yang PC, Yu CJ, Cj Y, et al. Interleukin-8 messenger ribonucleic acid expression correlates with tumor progression, tumor angiogenesis, patient survival, and timing of relapse in non-small-cell lung cancer. Am J Respir Crit Care Med. 2000;162(5):1957-1963.

25. Sunaga N, Kaira K, Tomizawa Y, et al. Clinicopathological and prognostic significance of interleukin-8 expression and its relationship to KRAS mutation in lung adenocarcinoma. $\mathrm{Br} J$ Cancer. 2014;110(8):2047-2053.

26. Desai S, Laskar S, Pandey BN. Autocrine IL-8 and VEGF mediate epithelial-mesenchymal transition and invasiveness via p38/JNKATF-2 signalling in A549 lung cancer cells. Cell Signal. 2013;25(9): 1780-1791.
27. Singh JK, Simões BM, Clarke RB, Bundred NJ. Targeting IL-8 signalling to inhibit breast cancer stem cell activity. Expert Opin Ther Targets. 2013;17(11):1235-1241.

28. Liotti F, Collina F, Pone E, et al. Interleukin-8, but not the related Chemokine CXCL1, sustains an autocrine circuit necessary for the properties and functions of thyroid cancer stem cells. Stem Cells. 2017;35(1): $135-146$.

29. Ginestier C, Liu S, Diebel ME, et al. CXCR1 blockade selectively targets human breast cancer stem cells in vitro and in xenografts. J Clin Invest. 2010;120(2):485-497.

\section{Publish your work in this journal}

OncoTargets and Therapy is an international, peer-reviewed, open access journal focusing on the pathological basis of all cancers, potential targets for therapy and treatment protocols employed to improve the management of cancer patients. The journal also focuses on the impact of management programs and new therapeutic agents and protocols on

\section{Dovepress}

patient perspectives such as quality of life, adherence and satisfaction. The manuscript management system is completely online and includes a very quick and fair peer-review system, which is all easy to use. Visit http://www.dovepress.com/testimonials.php to read real quotes from published authors.

Submit your manuscript here: http://www.dovepress.com/oncotargets-and-therapy-journal 